\title{
Proportional Fair Frequency-Domain Packet Scheduling for 3GPP LTE Uplink
}

\author{
Suk-Bok Lee* Ioannis Pefkianakis* \\ ${ }^{*}$ Computer Science Department \\ UCLA, CA 90095
}

\author{
Adam Meyerson* Shugong $\mathrm{Xu}^{\dagger} \quad$ Songwu $\mathrm{Lu}^{*}$ \\ ${ }^{\dagger}$ Huawei Technologies \\ Shanghai, China
}

\begin{abstract}
With the power consumption issue of mobile handset taken into account, Single-carrier FDMA (SC-FDMA) has been selected for 3GPP Long-Term Evolution (LTE) uplink multiple access scheme. Like in OFDMA downlink, it enables multiple users to be served simultaneously in uplink as well. However, its single carrier property requires that all the subcarriers allocated to a single user must be contiguous in frequency within each time slot. This contiguous allocation constraint limits the scheduling flexibility, and frequency-domain packet scheduling algorithms in such system need to incorporate this constraint while trying to maximize their own scheduling objectives.

In this paper we explore this fundamental problem of LTE SC-FDMA uplink scheduling by adopting the conventional timedomain Proportional Fair algorithm to maximize its objective (i.e. proportional fair criteria) in the frequency-domain setting. We show the NP-hardness of the frequency-domain scheduling problem under this contiguous allocation constraint and present a set of practical algorithms fine tuned to this problem. We demonstrate that competitive performance can be achieved in terms of system throughput as well as fairness perspective, which is evaluated using 3GPP LTE system model simulations.
\end{abstract}

\section{INTRODUCTION}

In recent years Orthogonal Frequency Division Multiple Access (OFDMA) has been considered as a strong candidate for the broadband air interface for its robustness to multipath fading, higher spectral efficiency and bandwidth scalability, and it has been selected for 3GPP Long-Term Evolution (LTE) downlink (DL) radio access technology. However, one major disadvantage of OFDMA is that the instantaneous transmitted RF power can vary dramatically within a single OFDM symbol. Such an undesirable high peak-to-average power ratio (PAPR) is a serious concern for the uplink (UL), since power consumption is a key consideration for the mobile handsets. As a result of seeking an alternative to OFDMA, Singlecarrier FDMA (SC-FDMA) has been selected for LTE uplink multiple access scheme. While keeping most of the advantages of OFDMA (e.g. the same degree of multipath protection), SCFDMA has significantly lower PAPR, since the underlying waveform is essentially single-carrier. Thus, lower PAPR of SC-FDMA greatly benefits the mobile terminal in terms of transmit power efficiency.

As in DL OFDMA, multiple access in UL SC-FDMA is achieved by assigning different frequency portions of the system bandwidth to individual users based on their channel conditions. Such simultaneous frequency-domain multiplexing of users (inherently in concert with time-domain scheduling) is performed by frequency domain packet scheduling (FDPS).

\footnotetext{
${ }^{\dagger}$ This work was performed when Shugong Xu was with Sharp Laboratories of America, where he supervised this work.
}

In LTE UL, the system bandwidth is divided into multiple subbands (i.e. groups of subcarriers) denoted as physical resource blocks (RBs). In order to achieve large gain from multiuser frequency diversity, a scheduler needs to know the instantaneous radio channel conditions across all users and all $\mathrm{RBs}$, which are fed as input for the frequency-domain adaptive user-to-RB allocation. For example, in LTE UL each user transmits a Sounding Reference Signal (SRS) to the scheduling node (i.e. base station) [1], which is used as channel quality indicator (CQI). With CQIs across all users and all RBs, a base station performs RB-to-user assignment at each time slot (e.g. in LTE every $1 \mathrm{~ms}$ ) according to the selected scheduling policy. Thus, in the time-frequency domain, an RB is considered as a minimum scheduling resolution, and also a minimum unit of the data-rate adaptation by adaptive modulation and coding $(\mathrm{AMC})$ with a granularity of one sub-frame.

Most of the DL FDPS algorithms proposed so far adopt the well-known time-domain Proportional Fair (PF) algorithm as a basic scheduling principle and apply the PF algorithm directly over each RB one-by-one independently. However, such scheduling strategies cannot be employed in the UL SCFDMA. Due to its single carrier property, SC-FDMA requires that all the RBs allocated to a single user must be contiguous in frequency within each time slot (i.e. sub-frame) [5], [6]. Thus, LTE UL FDPS algorithms should respect this constraint while trying to maximize their own scheduling objectives.

In this paper we study this fundamental problem of UL frequency-domain packet scheduling under contiguous RB allocation constraint. We analyze this problem by adopting the widely employed PF algorithm to maximize its objective (i.e. proportional fair criteria) in the frequency-domain setting. The main goal of this paper is to investigate how to adapt the time-domain PF algorithm to this problem framework.

\section{A. The Model}

We consider a cellular network whose UL system bandwidth is divided into $m$ RBs, and we have a single base station and $n$ active wireless users. The base station can allocate $m$ RBs to a set of $n$ users. At each time slot multiple RBs (with the contiguity constraint) can be assigned to a single user, each RB however can be assigned to at most one user. In this paper we shall work in an infinitely backlogged model in which for each user there is always data available for service. Thus, the base station can schedule all the $m$ RBs every time slot.

We define the indicator variable $x_{i}^{c}(t)$ to indicate whether or not $\mathrm{RB} c$ is assigned to user $i$ at time slot $t$. We assume that channel conditions vary across RBs as well as users. 
The channel conditions typically depends on the channel frequency, so they may be different for different channels; moreover, they also depends on the user location and the time slot. Therefore, each RB has user-dependent and time-varying channel condition. We use $r_{i}^{c}(t)$ to denote the instantaneous channel rate for user $i$ on $\mathrm{RB} c$ at time $t$. This channel rates are estimated from the CQIs extracted from the UL channel sounding. Thus, if $x_{i}^{c}(t)=1$, then user $i$ can transmit data of size $r_{i}^{c}(t)$ on RB $c$ at time slot $t$.

\section{B. Problem Formulation}

In the time-domain context, the well known Proportional Fair (PF) algorithm aims to maximize the logarithmic utility function $\sum_{i} \log R_{i}$, where $R_{i}$ is the long-term service rate of user $i$. This objective is known as proportional fair criteria. In order to maximize $\sum_{i} \log R_{i}$, one should maximize $\sum_{i} d_{i}(t) / R_{i}(t)$ where $d_{i}(t)$ is total data transmitted to user $i$ at time $t$ (proven in [7], [10], [14]). Hence the time-domain PF algorithm always serves the user who maximizes $r_{i}(t) / R_{i}(t)$ at each time step $t$. Note that the PF algorithm achieves high throughput and maintains proportional fairness among all users by giving priority to users with a high-quality channel rate $\left(r_{i}(t)\right)$ and a low current average service rate $\left(R_{i}(t)\right)$.

We now adapt this time-domain PF metric to the frequencydomain setting with the utility function $\sum_{i} \log R_{i}$ as our objective. Let $\lambda_{i}^{c}(t)=r_{i}^{c}(t) / R_{i}(t)$ be the $P F$ metric value that user $i$ has on RB $c$ at time slot $t$. We can establish a FDPS version of PF objective function when scheduling time slot $t$ as follows:

$$
\max \sum_{i} \sum_{c} x_{i}^{c}(t) \lambda_{i}^{c}(t)
$$

It is fairly straightforward to see that objective (1) maximizes $\sum_{i} d_{i}(t) / R_{i}(t)$ at time step $t$, and therefore achieves proportional fairness, i.e. optimizing objective (1) maximizes the utility function $\sum_{i} \log R_{i}$ in the time and frequency domain context. For this reason, most of the proposed DL FDPS scheduling algorithms apply the PF algorithm directly over each RB one-by-one, i.e. for RB $c$ the PF algorithm selects the best user who maximizes $r_{i}^{c}(t) / R_{i}(t)$ at time slot $t$. However, for LTE UL we need to incorporate the contiguous RB constraint into this objective (1) due to the physical layer requirement of SC-FDMA. The consequence is that we now cannot apply the PF algorithm on each RB one-by-one in isolation. In other words, the isolated local optimization of each RB hardly optimizes the objective (1). Figure 1 exemplifies the case. With the contiguity constraint

\begin{tabular}{|c|c|c|c|c|c|c|c|c|c|c|}
\hline & & & & & & & & & & \\
\hline ser & a & & & & & & & & & \\
\hline A & 8 & & 6 & & & & 4 & & 6 & 718 \\
\hline B & 1 & \begin{tabular}{|l|}
8 \\
\end{tabular} & 1 & 8 & 2 & 8 & & & & \begin{tabular}{l|l}
7 & 1
\end{tabular} \\
\hline C & 6 & 6 & 6 & 5 & 5 & 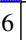 & & 4 & & \begin{tabular}{l|l}
6 & 5
\end{tabular} \\
\hline D & 3 & 4 & 5 & & & & 9 & & 7 & \begin{tabular}{l|l}
6 & 5
\end{tabular} \\
\hline E & 7 & 8 & 6 & 3 & 6 & 4 & 5 & 8 & 2 & 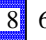 \\
\hline
\end{tabular}

\begin{tabular}{|c|c|c|c|c|c|c|c|c|c|c|c|}
\hline \multirow{2}{*}{\multicolumn{12}{|c|}{$\begin{array}{l}\text { w/ contiguous requirement } \\
\text { carrier } \quad \operatorname{Max}=83\end{array}$}} \\
\hline & & & & & & & & & & & \\
\hline \multicolumn{12}{|c|}{ 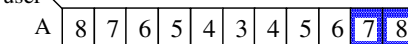 } \\
\hline \multicolumn{12}{|c|}{ 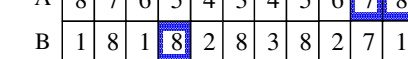 } \\
\hline $\mathrm{C}$ & 6 & 6 & 6 & 5 & 5 & & 4 & 4 & & & 5 \\
\hline & 3 & 4 & 5 & & & & & & & & 5 \\
\hline & & & & & & & & & & & 6 \\
\hline
\end{tabular}

Fig. 1 Maximizing the PF objective. The numbers denote the PF metric values $\lambda_{i}^{c}$. Dark-colored RBs represent assignment strategies maximizing the objective with/without the contiguity constraint. we may need to serve users with suboptimal PF metric value $\lambda_{i}^{c}$ for some RBs so as to optimize the PF objective (1).

Seeking to maximize the PF objective (1) under this contiguity constraint, we present four variations of PF-FDPS algorithm ( $A l g 1$ through $A l g 4)$. In this paper we explore the fundamental nature of this scheduling problem by investigating how well these algorithms fit into the problem framework.

\section{Hardness Result}

This contiguous RB allocation constraint is sufficient to make the problem hard.

Theorem 1: LTE UL PF-FDPS problem (i.e. maximizing objective (1) with the contiguous RB constraint) is NP-hard. The proof is omitted due to length constraints. It is presented in the full version of the paper [11].

\section{Heuristic Algorithms}

In this section we present a set of greedy heuristic algorithms for objective (1) under contiguous RB constraint. Our heuristics do not give guaranteed error bound, and moreover we believe that no practical greedy algorithms can give an approximation to this particular problem. ${ }^{1}$ Our heuristics finetuned to the typical instances of the problem might not perform well in their worst case scenarios, yet their overall performance is very good in practice, as shown in Section III.

\section{A. Alg1: carrier-by-carrier in turn}

As a starter, our first greedy heuristic $A l g 1$ schedules data from RB1 to RB $m$ in sequence, and for each $\mathrm{RB} c$ it assigns the best user $i$ who 1) has the maximum PF metric value $\lambda_{i}^{c}$ on $c$ and 2) satisfies the contiguity constraint.

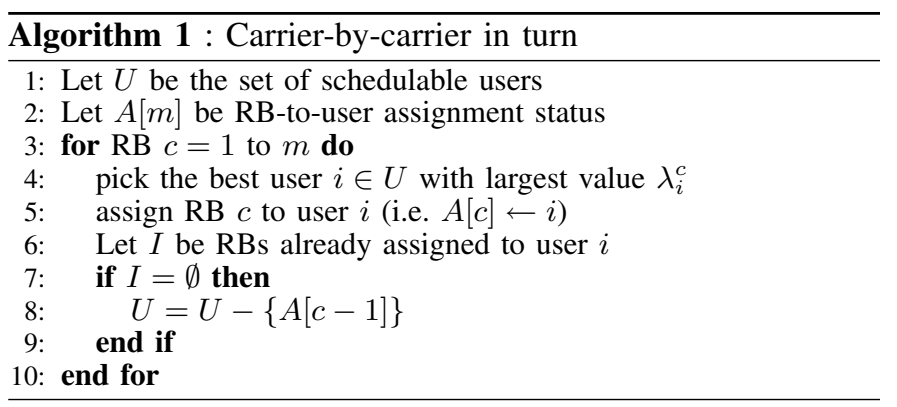

Since $A l g 1$ schedules data from one end side $\mathrm{RB}$, it is not likely to even have a chance to try users' high metric value frequency portions.

\section{B. Alg2: largest-metric-value-RB-first}

Viewing this scheduling problem as simply a packing problem, adhering to its rule of thumb "pack large items first" may help in our case. Adopting such a quite intuitive judgement, Alg2 schedules RBs with largest metric value first. However, it is uncertain how our action should be in the case that, for a certain user $i$ a candidate RB is not adjacent to RBs already assigned to $i$ (e.g. RB3 is first assigned to $i$, then the next largest value one is RB5 of $i$. If RB4 is already assigned to

${ }^{1}$ We developed a randomized algorithm that gives $\frac{1}{2}$-approximation for this problem, but is too complex to be employed for practical wireless scheduling. The algorithm is presented in the full version of the paper [11]. 


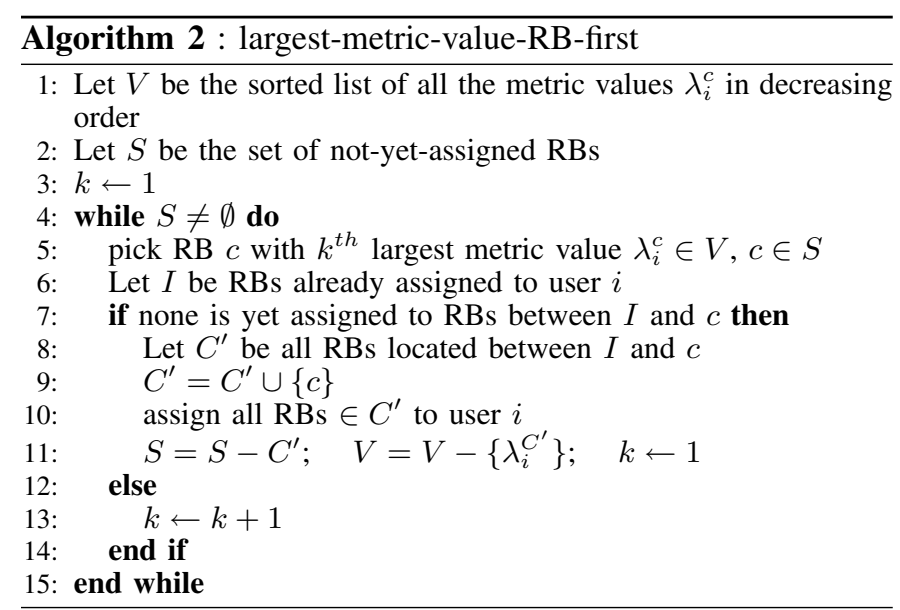

other user, then the contiguity constraint prohibits $i$ from being assigned to RB5. Should we however assign RB5 to $i$ if RB4 is still unoccupied?). Alg2 assigns those candidate RBs unless it violates the contiguity constraint (i.e. it assigns RB5 to $i$ ).

The price we pay for this a bit aggressive strategy is that we have to assign all the "in-between" RBs to a candidate user (i.e. it assigns RB5 to $i$, which as a result comes with assignment of RB4 to $i$, since $i$ is already assigned RB3). The downside of this approach comes from this by-product assignment. Since the length of such "in-between" RBs is arbitrary, a potential improvement in those RBs is likely to be cancelled.

\section{Alg3: riding peaks}

Seeing the drawback of $A l g 2$, we would like to utilize each user's high valued RBs as much as possible. Let's look at the PF metric values $\left(\lambda_{i}^{c}(t)=r_{i}^{c}(t) / R_{i}(t)\right)$ at time slot $t$. One key observation is that, for each user $i$ the denominator $\left(R_{i}(t)\right)$ is constant for all $\mathrm{RBs}$, so the resulting value for each RB $c$ is dominated by channel rate $\left(r_{i}^{c}(t)\right)$ only scaled down/up to the current service rate. Thus, at time slot $t$ each user's RB values fluctuate exactly as the channel rate changes between RB to RB. However, another fundamental physical layer characteristic is that in multi-carrier systems the channel SNR values (i.e. CQI) are correlated in both time and frequency (depending on the Doppler effect and the delay spread) [8], [12], [15]. In other words, if for each user $i \mathrm{RB} c$ has good channel rate, then the neighboring $\operatorname{RBs}(c-1, c+1)$ have high channel rate as well with high probability.

So the key idea of $A l g 3$ is to "ride users' peaks" in

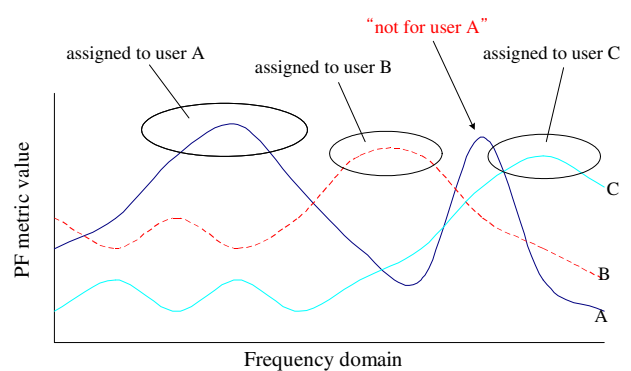

Fig. 2 Alg3 rides peaks.

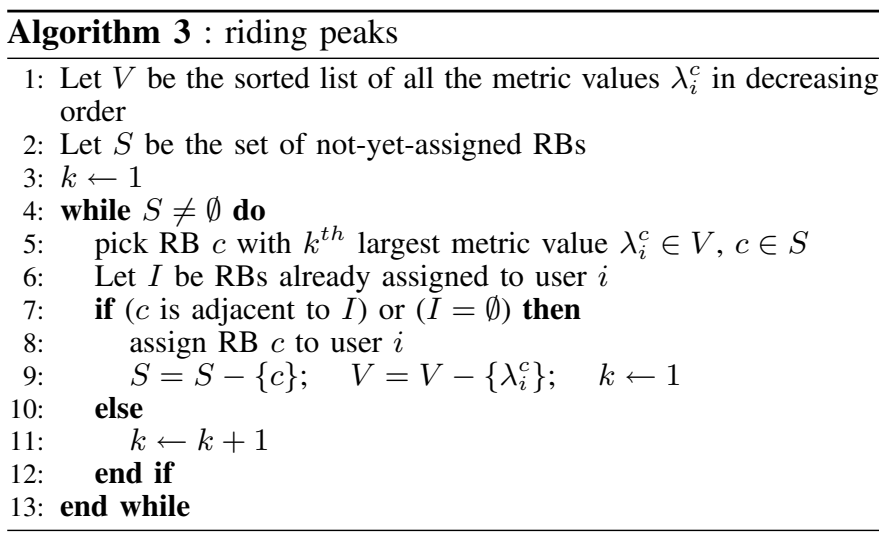

frequency domain, by exploiting such correlations. Recall that the conventional $\mathrm{PF}$ algorithm rides peaks in time domain. $A l g 3$, in fact, extends $A l g 2$ 's rule of thumb: 1) look at large value RBs first; 2) augment them by one neighbor RB. This second rule enforces a bit conservative contiguity condition (i.e. for a certain user $i$ a candidate RB must be adjacent to RBs already assigned to $i$ ).

Figure 2 illustrates the "peak riding" of $A l g 3$. In the beginning user $A$ is first assigned to its high value RBs, while user $B$ and $C$ are assigned to their peak RBs a little bit later. In the end they are all assigned to the RBs around their peaks according to the rules. Note that $A l g 2$ fails to allocate user $B$ to its high value RBs, since $B$ 's peak RB is surrounded by a bit higher $A$ 's peak RBs.

This "peak riding" approach so far seems quite good. There exist, of course the cases where it can lead to arbitrarily bad solutions. If for a certain user the channel rate across RBs changes arbitrarily, then sticking to peaks is not likely a good strategy. As mentioned earlier, we however can find typical instances displaying the frequency-domain correlation among $\mathrm{RBs}$, and in fact, this approach can lead to a measurable improvement on both throughput and short-term fairness in the realistic UL SC-FDMA scenarios as shown in Section III.

\section{Alg4: RB grouping}

Given that the frequency domain exhibits a correlation (more precisely, correlation between two adjacent RBs), Alg3 is expected to yield good performance. As mentioned in Section II-C, the channel quality values are indeed correlated in both time and frequency. However, in general the correlation in the frequency-domain is not as strong as the one in the timedomain (frequency-selective fading distortion) [12], [13]. That implies that we have the overall frequency correlation but its granularity may not be as small as one RB (i.e. the smooth lines in Figure 2 may need to be changed to the uneven ones). Figure 3 (overall fluctuation similar to Figure 2 but with some jitters) shows that such a condition incurs poor results by $A l g 3$. Since $A l g 3$ relies on the strong frequency-domain correlation, it is easily cheated by the small-scale variation. In the figure, user $B$ is falsely assigned to the abrupt peak, user $A$ is trapped by the sudden drop, and in the end user $C$ expands its region to that point.

To deal with such small-scale variation, it would help to extend our unit of consideration (i.e. the number of contiguous 


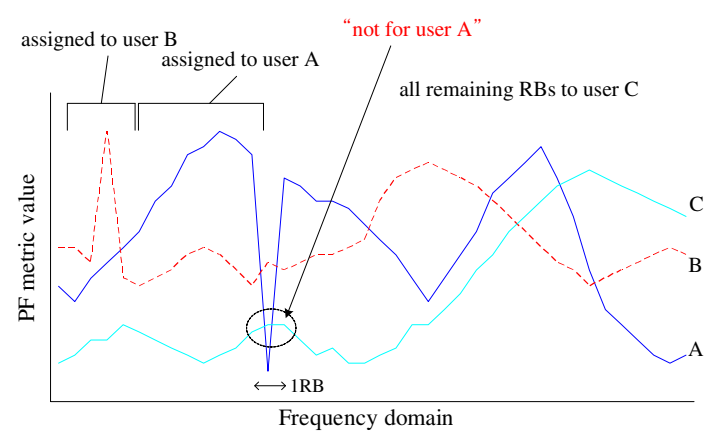

Fig. 3 Alg3 suffers from small-scale variation.

RBs that we view at a time). This RB grouping might be helpful to catch a bit large-scale fluctuation. Alg4 makes use of RB grouping to manage the weak frequency-domain correlation. The following questions may arise: "how big should a group be?", "is it a variable size?", and "freedom of positioning?". The harder we try to set up good criteria regarding those questions, it becomes more a quagmire due to the NP-hard nature. Here we set up simple rules: 1) divide $m$ RBs into $n$ groups; 2) apply the "peak riding" over those RB groups. Thus, Alg4 is an RB-grouping version of Alg3; Alg4 "rides peaks" with the granularity of RB groups (one group $=\left\lceil\frac{m}{n}\right\rceil$ RBs). Notice that as $n$ (i.e. the number of users) grows, the group size gets smaller (i.e. we see the smaller-scale fluctuation). As a ground for our choice of $\left\lceil\frac{m}{n}\right\rceil$, we argue that it would be beneficial to see the small-scale fluctuation with large number of users, since high multiuser frequency diversity can facilitate the potential improvement from the small-scale peaks.

One can easily find a bad example for $A l g 4$ and its inapproximability as well. However, such extremely bad instances are unlikely to happen in practice, and in fact, Alg4 exhibits constantly better performance over Alg3 on the real traces, particularly when the number of users is not large (as $n$ grows, $\left\lceil\frac{m}{n}\right\rceil$ RBs becomes $\left.1 \mathrm{RB}\right)$.

\section{SIMULATIONS}

To evaluate the performance of our heuristics, SC-FDMA uplink system level simulations have been conducted based on 3GPP LTE system model. We use traces generated as specified in 3GPP deployment evaluation [2], based on Typical Urban channel model. Table 1 summarizes a list of the default simulation parameters and assumptions.

We analyze the performance of the algorithms in terms of throughput as well as short-term fairness ${ }^{2}$, and assess how well they emulate the proportional fair criteria in this FDPS setting. However, since it is NP-hard to optimize objective (1) under the contiguity constraint, we do not have such an optimal algorithm in our hand. Thus, we use an algorithm that optimizes objective (1) without the constraint as our reference, and we refer to this algorithm as $O P T^{*}$. Note that $O P T^{*}$ offers an upper bound of the optimum. We use Jain's fairness index [9], measured by the data-rate fairness

\footnotetext{
${ }^{2} \mathrm{~A}$ well-known problem of the conventional time-domain PF scheduling is its poor short-term fairness.
}

TABLE I Simulation parameters

\begin{tabular}{l|l}
\hline Parameter & Setting \\
\hline System bandwidth & $20 \mathrm{MHz}$ \\
Subcarriers per RB & 12 \\
RB bandwidth & $180 \mathrm{kHz}$ \\
Number of RBs & 96 \\
Cell-level user distribution & Uniform \\
Number of active users in cell & $10,20,30,40,50$ \\
Traffic model & Infinitely backlogged \\
Transmission time interval (TTI) & $1 \mathrm{~ms}$ \\
Channel model & Typical Urban \\
User speed & $3,30,120 \mathrm{~km} / \mathrm{h}$ \\
User receiver & 1x2/MMSE/ZF \\
Modulation/coding rate settings & QPSK: $1 / 3,1 / 2,2 / 3,3 / 4$ \\
& $16 \mathrm{AM}: 1 / 2,2 / 3,3 / 4$ \\
HARQ model & Ideal chase combining \\
HARQ Aak/Nack delay & $8 \mathrm{~ms}$ \\
Max. number of HARQ retransmission & 3 \\
\hline
\end{tabular}

criterion $^{3}: F_{\phi}(\Delta t)=\left[\sum_{i=1}^{N} \phi_{i}(\Delta t)\right]^{2} /\left[N \cdot \sum_{i=1}^{N} \phi_{i}(\Delta t)^{2}\right]$, where $\phi_{i}(\Delta t)$ denotes the actual data-rate user $i$ achieved in time interval $\Delta t$, with $N$ users in the system.

We first measure the system throughput of our algorithms with varying the number of active users in the cell. As shown in Figure 4(a), Alg4 results in the highest throughput among our heuristics, followed by $A l g 3, A l g 2$, and $A l g 1$. This trend seems to match with our expectation, since Alg4 and Alg3 contain more advanced heuristic idea than the other two. In general, Alg3 performs better than $A l g 1$ and $A l g 2$ because Alg3 seeks to take advantage of each users' peak while both $A l g 1$ and $A l g 2$ are not so fine-tuned enough to effectively utilize multiuser frequency diversity. However, as seen from Figure 4(a), Alg3 displays the poor performance with small number of active users (e.g. when $n=10$, it yields even lower throughput than $A l g 1$ and $A l g 2$ ). Such a result shows the implication of the weak frequency-domain correlation, by which Alg3 is easily misled into bad solutions. On the other hand, Alg 4 contantly outperforms the other three algorithms in all scenarios. Alg4 deals with this small-scale variations by widening its view to $\left\lceil\frac{m}{n}\right\rceil$ RBs. In the case of small number of active users, $A l g 4$ expands the RB-group size, and it rides each users' aggregated peak by catching a bit large-scale fluctuation (it attains $84 \%$ of $O P T^{*}$ while $A l g 3$ gets $77 \%$.). As $n$ grows, Alg4 adaptively lessens the view so as to exploit the smallscale fluctuation, and its performance gets similar to Alg3 (when $n=50, A l g 4$ and $A l g 3$ reach $95 \%$ of $O P T^{*}$ while the other two get around 86\%). It is worth stressing again that $O P T^{*}$ does not represent the optimum of our objective but simply shows an upper bound of it, where the actual optimum lies between $A l g 4$ and $O P T^{*}$ in general.

We now evaluate the short-term fairness of our algorithms with varying the number of active users. Figure 5(a) shows the short-term data-rate fairness $F_{\phi}(\Delta t)$, in the cell of 30 active users, with extending the time interval window $\Delta t$ from 10 ms (i.e. $10 \mathrm{TTI}$ ) to $50 \mathrm{~ms}$. In this setting, Alg3 consistantly outperforms other algorithms in all intervals, followed by Alg4, Alg1, and Alg2. To understand why Alg3 provides better short-term fairness than others in this setting, we record

\footnotetext{
${ }^{3} F_{\phi}(\Delta t)=1$ implies that all users received equal data-rate within time $\Delta t$.
} 


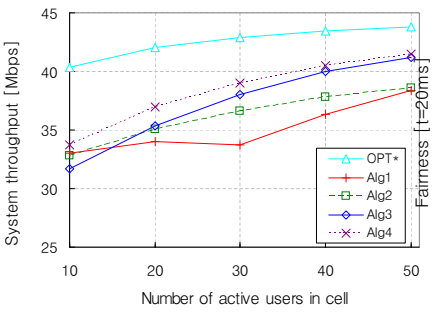

(a) cell throughput

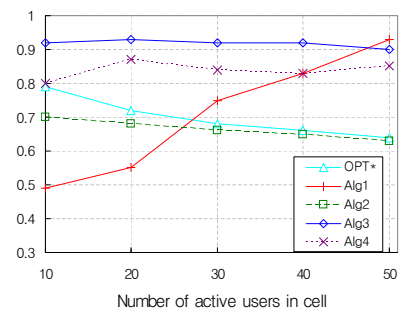

(b) fairness index $(\mathrm{t}=20 \mathrm{~ms})$
Fig. 4 System throughput and fairness with varying num. of users

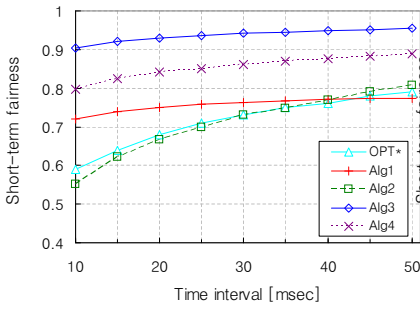

(a) 30 active user case

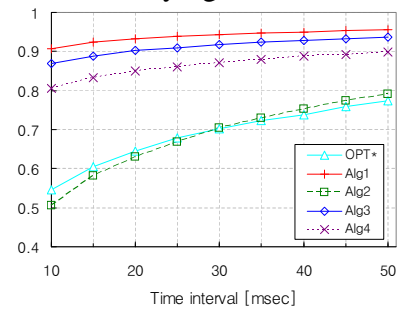

(b) 50 active user case
Fig. 5 Short-term fairness with varying time interval

the number of users scheduled per one TTI for each algorithm. Figure 6(a) plots the average number of users scheduled per one TTI when 30 users are active in the cell. We can see that all of 30 users are likely assigned to all 96 RBs by $A l g 3$ and Alg $1 .{ }^{4}$ However, the crucial difference is that $A l g 1$ is likely to allocate arbitrary rate on each user while $A l g 3$ seeks to assign users their peak RBs, which helps short-term "fair share" of the frequency resource. Figure 4(b) presents the short-term fairness of $20 \mathrm{~ms}$ interval window with increasing number of active users. Interestingly, $A l g 1$ offers the best fairness when the number of users is large (e.g. $n=50$ ). See also Figure 5 (b) and 6(b) for fairness and the average number of users scheduled per a TTI with 50 users. With the large number of users, $A l g 1$ is able to balance users' rates, but those are not likely from peak RBs.

At this point we need to compare the algorithms by a comprehensive metric that takes both throughput and fairness into account. Such a balance is pursued by the proportional fair criteria (i.e. maximizing $\sum_{i} \log R_{i}$, where $R_{i}$ is the long-term service rate for user $i$ ), which in fact is our ultimate objective function. Now we assess how well our heuristics emulate the proportional fair objective in our problem framework. In the following table we show the values of the PF criteria with 30 active users in the cell.

\begin{tabular}{|c|c|c|c|c|c|}
\hline & OPT $^{*}$ & Alg1 & Alg2 & Alg3 & Alg4 \\
\hline$\sum_{i} \log R_{i}$ & 223.1 & 216.5 & 218.9 & 220.6 & 221.6 \\
\hline
\end{tabular}

We can see that $A l g 4$ has the highest value of $\sum_{i} \log R_{i}$, followed by $A l g 3, A l g 2$ and $A l g 1$. We obtain the same trend (with similar gaps between values) in all other scenarios. As we underlined earlier, $O P T^{*}$ simply represents an upper bound of the optimum of our objective, so the actual optimum has a value of $\sum_{i} \log R_{i}$ between $A l g 4$ and $O P T^{*}$. Therefore,

\footnotetext{
${ }^{4}$ This result seems quite intuitive in the sense that $A l g 3$ and $A l g 1$ make assignment decision on one single RB at a time while Alg2 and Alg4 assign potentially multiple RBs to a certain user at a time.
}

(a) 30 active user case

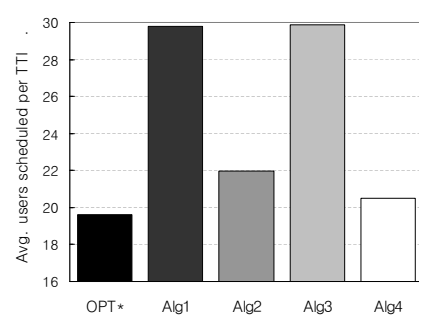

(b) 50 active user case

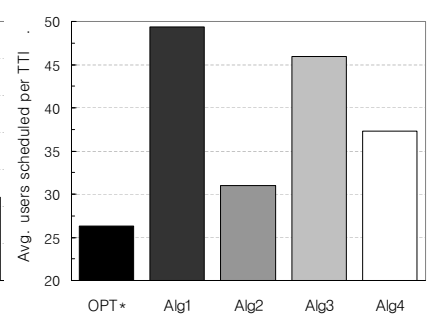

Fig. 6 Average num. of users scheduled per 1 TTI

among our heuristics $A l g 4$ has the value of the PF criteria closest to the actual optimum, and it emulates best the PF criteria in UL FDPS setting.

\section{Conclusions}

Due to its single carrier property of SC-FDMA, LTE UL requires the RBs allocated to a single user to be contiguous in frequency. In this paper we explored this fundamental problem of frequency-domain scheduling under contiguous RB allocation constraint. We investigated how to adapt the timedomain PF algorithm to this problem framework. We first showed the NP-hard nature of this problem, then presented a set of practical algorithms fine tuned to this problem. Among them, an algorithm that exploits the frequency-domain correlations in concert with an adaptive RB grouping technique emulates best the PF criteria in the LTE UL FDPS context.

Finally we believe that no practical wireless scheduling algorithms can give an approximation to this particular problem, but whether there actually exists such an algorithm or not still remains as an open problem.

\section{ACKNOWLEDGMENTS}

We thanks Prof. Adam Meyerson for his contribution in proving NP-hardness and developing an approximation algorithm for this problem.

\section{REFERENCES}

[1] Physical layer aspects for evolved Universal Terrestrial Radio Access (UTRA) 3GPP TR 25.814

[2] Technical specification group radio access networks - Deployment aspects. 3GPP TR 25.943

[3] Requirements for Evolved UTRA and UTRAN (Release 7). 3 GPP TR 25.913 V7.0.0, June 2005.

[4] TSG-RAN WG1 \#42, R1-050737, "Bandwidth of resource blocks for DL OFDMA", London, UK, Sep, 2005.

[5] Moray Rumney. 3GPP LTE: Introducing SIngle-Carrier FDMA Agilent Measurement Journal, 2008.

[6] 3GPP TSG-RAN WG2 Meeting \#57, R2-070585, "Resource fragmentation in LTE uplink", St. Louis, USA, Feb, 2007.

[7] M. Andrews. A survey of scheduling theory in wireless data networks. IMA, 2005

[8] E. Biglieri, J. Proakis, and S. Shamai. Fading channels: Information-theoretic and communications aspects. IEEE Trans. on Information Theory, 2000.

[9] R. Jain, D. M. Chiu, and W. Hawe. A Quantitative Measure of Fairness and Discrimination for Resource Allocation in Shared Systems. DEC Research Report TR-301.

[10] H. Kushner and P. Whiting. Asymptotic properties of proportional-fair sharing algorithms. Allerton, 2002.

[11] S.-B. Lee, I. Pefkianakis, A. Meyerson, S. Xu, and S. Lu. Proportional Fair Frequency-Domain Packet Scheduling for 3GPP LTE Uplink. UCLA TR-090001, 2009.

[12] B. Sklar. Rayleigh fading channels in mobile digital communication systems, Part I: Characterization. IEEE Communications Magazine, 1997.

[13] B. Sklar. Rayleigh fading channels in mobile digital communication systems, Part II: Mitigation. IEEE Communications Magazine, 1997.

[14] D. Tse. Multiuser diversity in wireless networks. http://www.eecs.berkeley.edu/ dtse/stanford416.ps , 2002.

[15] W. Wang, T. Ottosson, M. Sternad, A. Ahlen, and A. Svensson. Impact of multiuser diversity and channel variability on adaptive OFDM. IEEE VTC, 2003. 